\title{
The influence of solar wind turbulence on geomagnetic activity
}

\author{
D. Jankovičová ${ }^{1}$, Z. Vörös ${ }^{1,2}$, and J. Šimkanin ${ }^{3}$ \\ ${ }^{1}$ Institute of Atmospheric Physics, Academy of Sciences of the Czech Republic, Prague, Czech Republic \\ ${ }^{2}$ Space Research Institute, Austrian Academy of Science, Graz, Austria \\ ${ }^{3}$ Geophysical Institute, Academy of Sciences of the Czech Republic, Prague, Czech Republic
}

Received: 21 September 2007 - Revised: 22 November 2007 - Accepted: 20 December 2007 - Published: 5 February 2008

\begin{abstract}
The importance of space weather and its forecasting is growing as interest in studying geoeffective processes in the Sun - solar wind - magnetosphere - ionosphere coupled system is increasing. In this paper higher order statistical moments of interplanetary magnetic field and geomagnetic SYM-H index fluctuations are compared. The proper description of fluctuations in the solar wind can elucidate important aspects of the geoeffectivity of upstream turbulence and contribute to our understanding of space weather. Our results indicate that quasi-stationary intervals during both quiet and stormy periods have to be investigated in order to find correlations between upstream and geomagnetic conditions. We found that the fourth statistical moment (kurtosis), which was not considered in previous studies, appears to be a new geoeffective parameter. Intermittency of the magnetic turbulence in the solar wind can influence the efficiency of the solar wind - magnetosphere coupling through affecting magnetic reconnection at the Earth's magnetopause.
\end{abstract}

\section{Introduction}

Recent results in understanding the dynamical processes within the Sun - solar wind - magnetosphere - ionosphere coupled system have brought new questions in this field. All events at the Sun leading to large perturbations in the Earth's magnetosphere - ionosphere system are called geoeffective. As the importance of space weather and its forecasting grows, so does interest in studying geoeffective processes in this coupled system. The significance of the reconnection processes during periods of southward interplanetary magnetic field in exchanges of mass, energy and momentum between solar wind and magnetosphere plasmas, is well-known (e.g. Baker et al., 1996; Borovsky and Funsten,

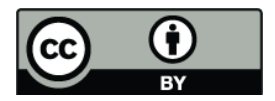

Correspondence to: D. Jankovičová (dja@ufa.cas.cz) 2003a,b). These exchanges consequently cause geomagnetic storms and substorms and the exchanges further continue with the Earth's ionosphere. Questions about fluctuations and their role in these processes have recently arisen. The solar wind is an inhomogeneous, anisotropic and compressible magnetized plasma, in which the bulk plasma parameters and the magnetic field fluctuate over a broad range of spatial and temporal scales (Tu and Marsch, 1995; Horbury and Tsurutani, 2001). It is a plasma flow originating from the Sun, showing typical turbulent and intermittent properties resembling those in laboratory turbulent flows (e.g. Zweben et al., 1979; Zank and Matthaeus, 1992; Oughton , 1993). These fluctuations were not usually taken into account in many past models. Recently, the two-component model of MHD fluctuations was proposed. This model considers an eddy turbulence (so-called 2-D-turbulence) in the perpendicular direction to the mean magnetic field $\mathbf{B}_{\mathbf{0}}$ together with so-called slab turbulence in the parallel direction to $\mathbf{B}_{\mathbf{0}}$ (presenting the Alfvén waves). In plasma, turbulence cannot exist without contemporary existence of outward- and inward- propagating Alfvén waves, which have to be decorrelated with each other (e.g. Bruno and Carbone, 2005).

Recently, more and longer term data sets have become available with better time resolutions. This makes it possible to do new studies of changes in fluctuation statistics with $11 \mathrm{yr}$ of solar activity cycle. The results differ depending on whether the fast or slow solar winds were considered (e.g. Tu and Marsch, 1995; Bruno and Carbone, 2005). The fast and slow winds have a different nature and their appearance also depends on the phase of the solar cycle (Bruno and Carbone, 2005). During the maximum of the solar activity cycle the fast wind comes from the polar parts of the Sun within the frame of the open magnetic field line regions identified by polar holes. In contrast, the slow wind appears in equatorial plane leaking from closed magnetic field line coronal features called "helmets". The situation greatly changes in the minimum phase of the solar activity cycle. The polar coronal holes, which during the maximum of activity are around

Published by Copernicus Publications on behalf of the European Geosciences Union and the American Geophysical Union. 


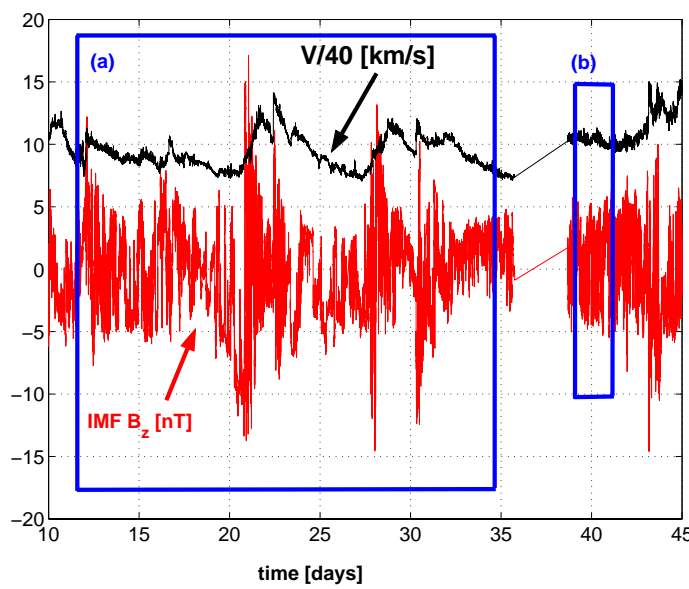

Fig. 1. Time behaviour of interplanetary magnetic field component $B_{z}$ (red) and the solar wind plasma velocity amplitude $|\mathbf{v}|$ (black). Example of (a) non-stationary interval containing mix of various physical situations; (b) more stationary interval proper for analysis of geoeffective properties.

the solar poles, reach the equatorial regions in minimum (Forsyth et al., 1997; Forsyth and Breen, 2002; Balogh et al., 1999). This new configuration produces an alternation of the fast and slow wind streams in the ecliptic plane. During the expansion, dynamical interactions develop between such fast and slow winds, generating so-called "stream interface", a thin region ahead of the fast stream characterized by strong compressive phenomena (Bavassano et al., 1997; Bruno and Carbone, 2005). The fast wind is less dense but hotter than slow wind and its proton number density and magnetic field intensity are more steady. The magnetic field vector of the fast wind fluctuates in direction much more than in the slow wind. It supports the idea that strong Alfvénic fluctuations are present, which mainly act on magnetic field and velocity direction vectors and are typical for the fast wind (e.g. Belcher and Solodyna, 1975). Within the slow wind, compressive regions can also appear, which precede the stream interface. However, they are not due to dynamical effects but by presence of the heliospheric current sheet between two opposite polarities of the interplanetary magnetic field (Bavassano et al., 1997).

The geoeffective role of Alfvénic fluctuations and magnetic structures was discussed in e.g. D'Amicis et al. (2007). They showed that the situation greatly changes during the solar activity cycle. While the Alfvénic fluctuations are geoeffective at the solar minimum phase, the magnetic structures play a role at the solar maximum phase. Chian et al. (2006) among others, also suggested that a close correlation between interplanetary Alfvén waves and AE index exists.

Increases in the number of spacecrafts, advances in techniques for observing the Sun and solar wind and in processing their datasets, allow much more advanced identification and better understanding of solar disturbances headed toward the Earth. All these possibilities together with better models of fluctuations can help in refining predictive models of magnetospheric disturbances e.g. magnetic storms and substorms, which represent a basic dynamic process in the magnetosphere (e.g. Jankovičová et al., 2002; Vörös and Jankovičová, 2002).

In this paper we are interested in role of the fluctuations in plasma processes within the solar wind - magnetosphere coupled system. We ask whether these fluctuations play any role in geoeffective processes besides all these well-defined geoeffective parameters and conditions studied earlier (e.g. Burton et al., 1975; Vassiliadis et al., 1993; O'Brien and McPherron, 2000). We examined the changes in the higher order statistical properties, namely the 3rd and 4th orders, of the solar wind and magnetospheric plasma data. We chose simultaneous data sets of the interplanetary magnetic field GSM component $B_{z}$ and SYM-H index, as a proper measure of the ring current intensity.

\section{Methods of analysis}

To be able to evaluate the fluctuation statistics of turbulent flows in plasma at different time scales, $\tau$, it is necessary to define the two-point differences, $\delta \boldsymbol{B}_{\tau}$, called increments (Frisch, 1995):

$\delta \boldsymbol{B}_{\tau}=\boldsymbol{B}(t+\tau)-\boldsymbol{B}(t)$.

It takes into account the Taylor hypothesis, which is well verified for solar wind (Bruno and Carbone, 2005). Turbulent plasma flows are usually described by the probability distribution function (PDF) of the field increments of velocity $\delta \boldsymbol{v}_{\tau}$ and magnetic field $\delta \boldsymbol{B}_{\tau}$ over the varying time scale $\tau$ (Frisch, 1995). In solar wind plasma the changes of shape of PDF with time scale $\tau$ have typical properties of intermittent behaviour in $\delta \boldsymbol{v}_{\tau}$ and $\delta \boldsymbol{B}_{\tau}$ (Sorriso-Valvo et al., 1999). At the larger scales $\tau$ it is nearly Gaussian but as the scale decreases the wings of the PDF become stretched due to the fact that large deviations from the average value of the fields increments are present.

Mathematically, it is necessary to know the infinite number of statistical moments to describe the PDF properly. In Gaussian case the first moment called mean value $\bar{x}$ and the second moment called variance $\sigma^{2}$ (or its squared root as the standard deviation $\sigma$ ) is enough to characterize it properly. But in the non-Gaussian case, the 2nd order statistics are not sufficient for the complete definition of PDF. It is necessary to take into account higher order statistics. In this paper we will deal with the third and fourth moments known as skewness and kurtosis. Skewness $(S)$ is the third standardized moment of a PDF and indicates the degree of asymmetry of a distribution

$$
S=\frac{1}{N} \sum_{j=1}^{N}\left[\frac{x_{j}-\bar{x}}{\sigma}\right]^{3} \text {. }
$$


(a)
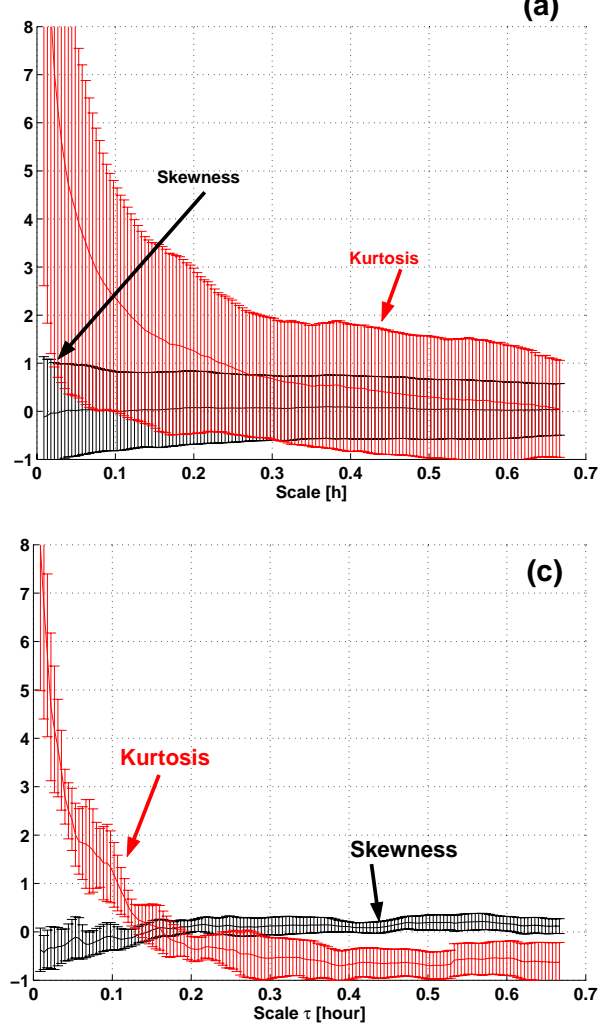

(b)
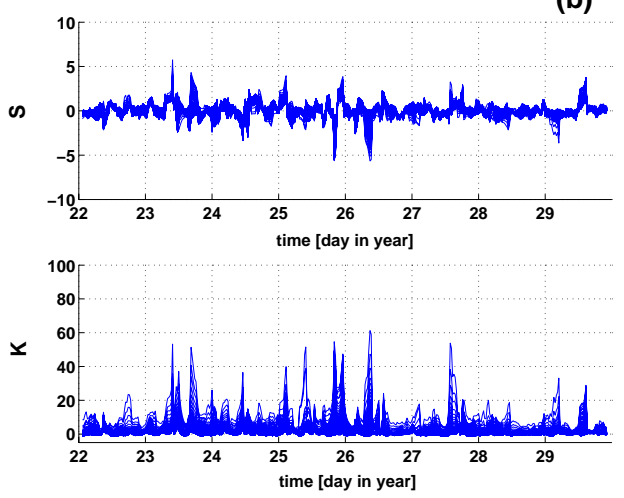

(d)
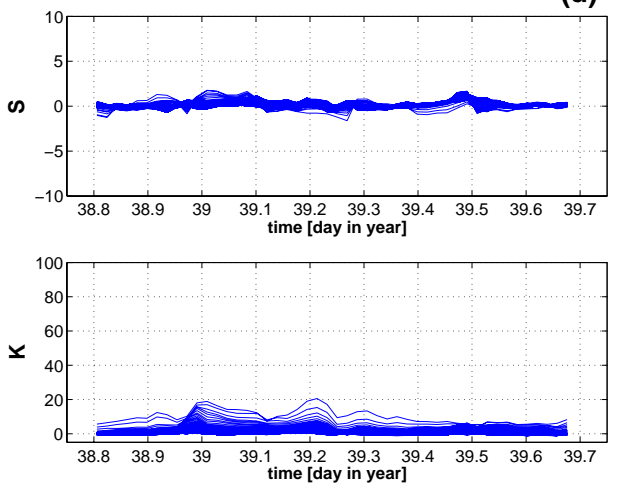

Fig. 2. (a,c) Skewness (black) and kurtosis (red) of the interplanetary magnetic field $B_{z}$ component vs time scales $\tau$ sketched for $\tau \in\langle 1$ min, $40 \mathrm{~min}\rangle$ for: (a) long non-stationary interval (see Fig. 1a), (c) stationary interval (see Fig. 1b). $S$ and $K$ were calculated within multiple shifted windows to be able to estimate the error bars. (b,d) The estimation of stationarity was done statistically, for different values of $\tau \in$ $\langle 1 \mathrm{~min} ; 40 \mathrm{~min}\rangle$ calculated for: (b) long non-stationary interval (see Fig. 1a), (d) stationary interval (see Fig. 1b).

If a distribution has a positive skewness the right tail is longer, otherwise skewness is negative if the left tail is longer. The skewness for Gaussian distribution is $S=0$. Kurtosis is the fourth standardized moment of a probability distribution PDF and presents the degree of peakedness of a distribution

$K=\left\{\frac{1}{N} \sum_{j=1}^{N}\left[\frac{x_{j}-\bar{x}}{\sigma}\right]^{4}\right\}-3$.

Positive kurtosis indicates a "peaked" distribution and negative kurtosis indicates a "flat" distribution. The Gaussian distribution has kurtosis $K=0$.

\section{Results}

For studying the geoeffective processes it was necessary to find simultaneous interplanetary and geomagnetic data sets. To this end the solar wind data was time shifted from the solar wind satellite position to the Earth using the solar wind convection velocity. For our analysis the time series of $16 \mathrm{~s}$ averages of interplanetary magnetic field $B_{z}$ (GSM) component measurements performed by ACE satellite during years
2000-2001 were used. As magnetospheric characteristics the SYM-H index with $\Delta \tau=1 \mathrm{~min}$ were chosen. SYM-H index is ideally regarded as a measure of the magnetospheric ring current intensity.

The raw data was transformed in several steps to create the basic database for our investigation. In the first step we differenced the data by Eq. (1). This equation represents a kind of high pass filtering process necessary to set off the fluctuations in the lower frequency range.

In the second step it was necessary to find proper periods for our analysis. In Fig. 1 it can be seen that real data sets are a mix of many physical situations and if very long periods would be analysed (Fig. 1a) it would not be possible to characterize particular processes leading to any response on magnetospheric side. Besides, such long intervals are nonstationary in multiscale sense (Fig. 2b) and they will give statistical results very far from the truth (Fig. 2a). For the sake of it, it was necessary to choose shorter (Fig. 1b) and more stationary data intervals (Fig. 2d), which would contain more simple physical situations. $S$ and $K$ were calculated with sliding window analysis within $\tau \in\langle 1 \mathrm{~min} ; 40 \mathrm{~min}\rangle$, which allowed to estimate the error bars (Fig. 2a,c). Afterwards, 
(a) CASE A

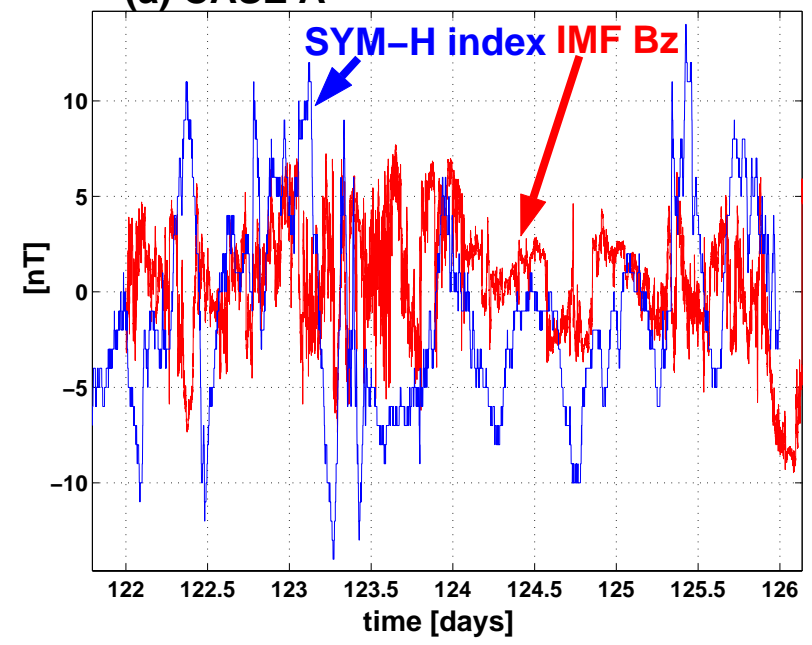

(b) CASE B

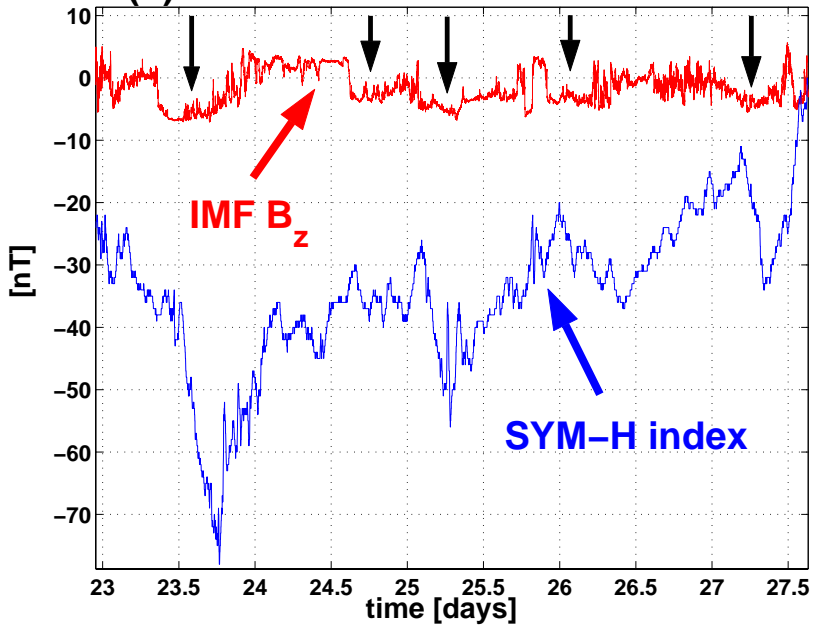

Fig. 3. Periods selected by special conditions described in detail in text: (a) CASE A (red - the interplanetary magnetic field $B_{z}$ component; blue - the magnetospheric SYM-H index) and (b) CASE B (black arrows $-B_{z}$ turning to $B_{z}<0$ for more than $2 \mathrm{~h}$ ).

the time evolutions of $S$ and $K$ for different values of time scales $\tau \in\langle 1 \mathrm{~min} ; 40 \mathrm{~min}\rangle$ were monitored (Fig. $2 \mathrm{~b}$,d). Consequently, only those data intervals were chosen for further analysis for which $S$ and $K$ could be estimated within sliding windows with small variability. We find that the stationarity of mean or standard deviation does not guarantee the stationarity of higher order moments for non-Gaussian dynamical processes. Fig. 2a shows the scale evolution of $S$ and $K$ for a longer (eight days) interval depicted in Fig. 2b. The estimated error bars indicate that multiple physical processes are present and the moments cannot be calculated straightforwardly. On the contrary, during shorter intervals the plasma parameters and the magnetic field do not change so much, therefore, $S$ and $K$ can be estimated more accurately. Fig. 2c shows the estimation of the scale dependency of moments for a quasi-stationary interval depicted in Fig. 2d.

Following these conditions, 54 intervals were chosen, which were divided into two groups, depending on conditions in the interplanetary magnetic field $B_{z}$ component and SYM-H index:

1. CASE A - 22 intervals (Fig. 3a)
(a) $\left|B_{z}\right|<10 \mathrm{nT}$
(b) $|\mathrm{SYM}-\mathrm{H}|<20 \mathrm{nT}$

2. CASE B - 32 intervals (Fig. 3b)

(a) $B_{z}$ turning to $B_{z}<0 \mathrm{nT}$ for more than $2 \mathrm{~h}$

(b) $\mathrm{SYM}-\mathrm{H}<-20 \mathrm{nT}$

The CASE A intervals were considered to be quiet time periods and CASE B as periods, which can lead to magnetospheric disturbances, observed in the SYM-H index.
The intervals contain one-day data (i.e. 5400 points), which were chosen not to contain interplanetary shocks. Obviously, longer intervals could contain more data points, but the available number of quasi-stationary intervals would be significantly reduced. In each of these 54 intervals the higher order moments $S$ and $K$ defined by Eqs. (2) and (3) were calculated in the moving window of $\mathrm{W}=45 \mathrm{~min}$ for different time scales $\tau \in\langle 1 \mathrm{~min}, 60 \mathrm{~min}\rangle$ (Fig. 4a-d). Figure 4a,b show no difference in the scale dependency of the skewness. The estimated values of $S$ fluctuate around zero for both the interplanetary magnetic field $B_{z}$ component and SYM-H index and this behaviour is independent on the selection criteria (CASES A or B). It means that the asymmetry of the probability density function does not appear to be a geoeffective key parameter. The scale dependency of kurtosis estimated from the interplanetary magnetic field $B_{z}$ component and SYM-H index for both CASES A and B is depicted in Fig. 4c,d. The increase of $K$ towards smaller scales is a sign of peaked distributions and intermittency. Intermittency is stronger in the solar wind than in the magnetosphere. However, quiet time periods (CASE A) exhibit smaller values of kurtosis than magnetically disturbed periods (CASE B) (Fig. 4c,d). Therefore, we consider the distinct scale evolution of the kurtosis to be an important physical parameter describing certain geoeffective features of the magnetic fluctuations in the solar wind.

The scatter plots of the $S$ and $K$ estimated from the interplanetary magnetic field $B_{z}$ component and SYM-H index are shown in Fig. 5 in order to demonstrate better the relationship between the solar wind magnetic fluctuations and SYM-H index fluctuations. The results for both cases A (black) and B (red) and the evolution of this relationship can be compared for different time scales $\tau_{1}=1 \min (\bullet)$, $\tau_{2}=10 \mathrm{~min}(\mathrm{o})$ and $\tau_{3}=20 \mathrm{~min}(\triangle)$. Again, the scatter plots 

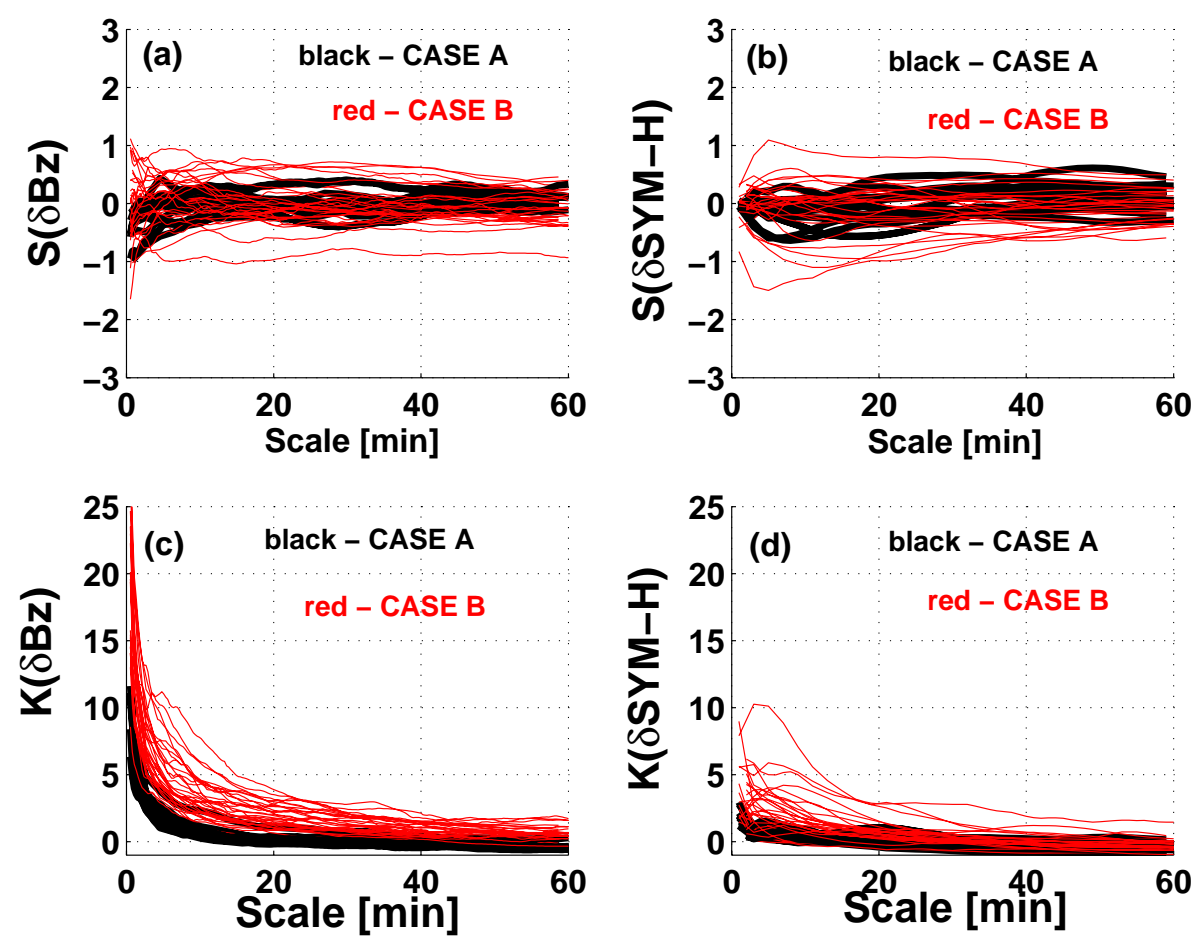

Fig. 4. The time scale $\tau$ dependence of (a,c) skewness $S$ and kurtosis $K$ of the interplanetary magnetic field component $B_{z}$ for time scales between $\tau \in\langle 16 \mathrm{~s}, 60 \mathrm{~min}\rangle$ in moving window $W=45 \mathrm{~min}$ for CASE A (black) and CASE B (red); (b,d) skewness $S$ and kurtosis $K$ of the magnetospheric SYM-H index for time scales between $\tau \in\langle 1 \mathrm{~min}, 60 \mathrm{~min}\rangle$ in moving window $W=45 \mathrm{~min}$ for CASE A (black) and CASE B (red).

of $S$ over different scales (Fig. 5a,c) show no clear patterns, while the scatter plots of $K$ over the same scales (Fig. 5b,d) show an interrelationship between the solar wind and magnetospheric fluctuations. Namely, larger values of kurtosis in the solar wind are associated with larger values of kurtosis in the magnetosphere and this relationship is visible at the scales of a few minutes.

We can suppose that magnetic fluctuations exhibiting large values of kurtosis or high intermittency in the solar wind might influence reconnection processes at the magnetopause. These results support the idea that at least part of the fluctuations observed at Lagrange point $L_{1}$, where ACE satellite operates, will reach the magnetosheath unchanged and can be supposed as a driver for the magnetosheath fluctuations, which consequently allow them to pass modified through the shock. Therefore, these features of the magnetic field fluctuations seem to represent specific conditions in the solar wind influencing the effectiveness of the solar wind - magnetosphere coupling.

\section{Conclusions}

It is well known that specific space weather conditions and an enhanced geomagnetic response strongly depend on the occurrence of geoeffective physical processes in the solar wind e.g. long lasting southward interplanetary magnetic field conditions lead to the appearance of strong geomagnetic storms. In this paper we studied the effect of interplanetary magnetic field fluctuations on the geomagnetic response represented by the SYM-H index. We examined the multi-scale evolution of higher order statistical properties in the solar wind and magnetospheric fluctuations. Quasi-stationary, simultaneous data sets of the interplanetary magnetic field $B_{z}$ component and the SYM-H index were chosen during both quite and active periods. Our results indicate that:

(i) The fluctuations of the solar wind cannot be always described as stationary random processes, because the fast and slow solar winds have different sources on the Sun. Therefore, different physical processes drive the fluctuations. From the point of view of consistent time series analysis and straightforward identification of specific processes in the solar wind, quasi-stationary intervals must always be selected for statistical estimations.

(ii) The asymmetry of the probability density functions described in terms of the skewness does not seem to be important as a geoeffective parameter. 

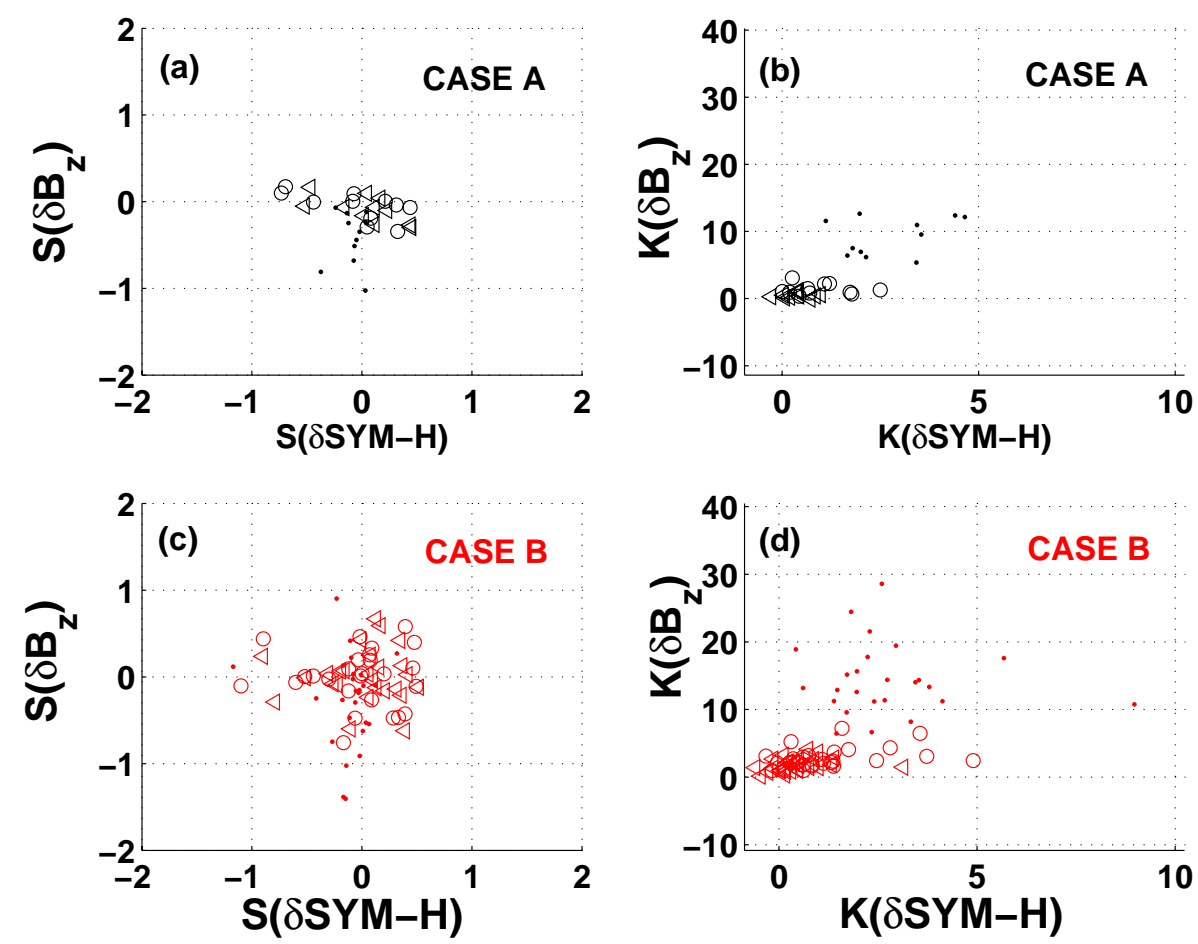

Fig. 5. Scatter plots of the skewness $S$ of the magnetospheric SYM-H index vs skewness of the inteplanetary magnetic field component $B_{z}$ for (a) CASE A and (c) CASE B; and the scatter plots of the kurtosis $K$ of the magnetospheric SYM-H index vs kurtosis of the inteplanetary magnetic field component $B_{z}$ for (b) CASE A and (d) CASE B computed for different time scales $\tau_{1}=1 \mathrm{~min}(\bullet), \tau_{2}=10 \mathrm{~min}(\mathrm{o})$ and $\tau_{3}=20 \mathrm{~min}$ $(\triangle)$.

(iii) The increase of kurtosis (Fig. 4b,d) towards small scales is representative in the interplanetary magnetic field $B_{z}$ component and SYM-H index time series and differs for quiet and more active periods. Thus, we can assume that the kurtosis estimated from solar wind magnetic fluctuations appears to be a representative geoeffective parameter, which can influence reconnection processes at the Earth's magnetopause and the efficiency of the solar wind - magnetosphere coupling.

Acknowledgements. This research was supported by the Grant Agency of the Academy of Sciences of the Czech Republic (Grant No. B300420509) and the INTAS Foundation (Grant 06-1000017-8943). We would like to thank for ACE database to the NOAA Space Environment Center and to the World data center for Geomagnetism, Kyoto, Japan for geomagnetic the SYM-H index data. We would like to thank very much S. Saxonberg and L. Sorriso-Valvo for their comments, which significantly improved our paper.

Edited by:P.-L. Sulem

Reviewed by:Two anonymous referees

\section{References}

Baker, D. N., Pulkkinen, T. I., Angelopoulos, V., Baumjohann, W., and McPherron, R. L.: The neutral line model of substorms: Past results and present view, J. Geophys. Res., 101(A6), 12975$13010,1996$.

Balogh, A., Forsyth, R. J., Lucek, E. A., Horbury, T. S., and Smith, E. J.: Heliospheric magnetic field polarity inversions at high heliographic latitudes, Geophys. Res. Lett., 26, 631-634, 1999.

Bavassano, B., Woo, R., and Bruno, R.: Heliospheric plasma sheet and coronal streamers, Geophys. Res. Lett., 24, 1655-1658, 1997.

Belcher, J. W. and Solodyna, C. V.: Alfvén waves and directional discontinuities in the interplanetary medium, J. Geophys. Res., 80, 181-186, 1975.

Borovsky, J. E. and Funsten, H. O.: Role of solar wind turbulence in the coupling of the solar wind to the Earth's magnetosphere, J. Geophys. Res., 108(A6), 1246, doi:10.1029/2002JA009601, 2003 (referred as 2003a).

Borovsky, J. E. and Funsten, H. O.: MHD turbulence in the Earth's plasma sheet: Dynamics, dissipation and driving, J. Geophys. Res., 108(A7), 1284, doi:10.1029/2002JA009625, 2003 (referred as 2003b).

Bruno, R. and Carbone, V.: The solar wind as a turbulence laboratory, Living Rev. Solar Phys., 4, 1-186, 2005.

Burton, R. K., McPherron, R. L., and Russell, T. C.: An empirical relationship between interplanetary conditions and Dst, J. Geo- 
phys. Res., 80(31), 4204-4214, 1975.

Chian, A. C., Kamide, L. Y., Rempel, E. L., and Santana, W. M.: On the chaotic nature of solar-terrestrial environment: Interplanetary Alfvén intermittency, J. Geophys. Res., 111, A07S03, doi:10.1029/2005JA011396, 2006.

D'Amicis, R., Bruno, R., and Bavassano, B.: Is geomagnetic activity driven by solar wind turbulence?, Geophys. Res. Lett., 34, L05108, doi:10.1029/2006GL028896, 2007.

Forsyth, F. J., Balogh, A., Horbury, T. S. and Smith, E. J.: The heliospheric magnetic field at solar minimum as observed by ULYSSES, Adv. Space Res., 19, 839-842, 1997.

Forsyth, B. and Breen, A.: The 3D Sun and heliosphere at solar maximum, meeting report, Astron. Geophys., 43(3), p. 3.32., doi:10.1046/j.1468-4004.2002.43332.x, 2002.

Frisch, U.: Turbulence: The legacy of A.N. Kolmogorov, Cambridge University Press, Cambridge, UK; New York, USA, 1995.

Horbury, T. S. and Tsurutani, B.: Ulysses measurements of waves, turbulence and discontinuities, in: The Heliosphere Near Solar Minimum: The Ulysses perspective, edited by: Balogh, A., Marsden, R. G., and Smith, E. J., Springer-Praxis Books in Astrophysics and Astronomy, Springer, London, UK; New York, USA, 167-227, 2001.

Jankovičová, D., Dolinský, P., Valach, F., and Vörös, Z.: Neural network based nonlinear prediction of magnetic storms, J. Atmos. Sol.-Terr. Phys., 64, 651-656, 2002.
O'Brien, T. P. and McPherron, R. L.: An empirical phase space analysis of ring current dynamics: Solar wind control of injection and decay, J. Geophys. Res., 105(A4), 7707-7719, 2000.

Oughton, S., Transport of solar wind fluctuations: A turbulence approach, PhD Thesis, Delaware University, Wilmington, USA, 1993.

Sorriso-Valvo, L., Carbone, V., Veltri, P., Consolini, G., and Bruno, R.: Intermittency in the solar wind turbulence through probability distribution functions of fluctuations, Geophys. Res. Lett., 26, 1801-1804, 1999.

Tu, C. Y. and Marsch, E.: MHD structures, waves and turbulence in the solar wind: observations and theories, Space Sci. Rev., 73, 1-210, 1995.

Vassiliadis, D., Sharma, A. S., and Papadopoulos, K.: An empirical model relating the auroral geomagnetic activity to the interplanetary magnetic field, Geophys. Res. Lett., 20, 1731-1734, 1993.

Vörös, Z. and Jankovičová, D.: Neural network prediction of geomagnetic activity: a method using local Hölder exponent, Nonlin. Processes Geophys., 9, 425-433, 2002, http://www.nonlin-processes-geophys.net/9/425/2002/.

Zank, G. P. and Matthaeus, W. H.: Waves and turbulence in the solar wind, J.Geophys. Res., 97, 17189-17194, 1992.

Zweben, S. J., Manyuk, C. R. and Taylor, R. J.: Small-Scale Magnetic Fluctuations Inside the Macrotor Tokamak, Phys. Rev. Lett., 42, p. 1720, doi: 10.1103/PhysRevLett.42.1720.3, 1979. 